\title{
Effect of cognitive task on components of 7 meter timed up-and-go test in persons with stroke
}

\author{
Nithinun Chaikeeree $^{\mathrm{a}}$, Butsara Chinsongkram ${ }^{\mathrm{b}}$, Vitoon Saengsirisuwan ${ }^{\mathrm{c}}$, Rumpa Boonsinsukh ${ }^{\mathrm{a}, *}$ \\ a Division of Physical Therapy, Faculty of Physical Therapy, Srinakharinwirot University, \\ Nakhon Nayok 26120 Thailand \\ b Faculty of Physical Therapy, Rangsit University, Pathum Thani 12000 Thailand \\ c Department of Physiology, Faculty of Science, Mahidol University, Rama 6 Road, \\ Bangkok 10400 Thailand
}

*Corresponding author, e-mail: rumpa@g.swu.ac.th

Received 20 Oct 2017

Accepted 28 Aug 2018

\begin{abstract}
A problem dealing with dual task is commonly found in persons post-stroke but it is unclear whether age and educational levels influence this performance. The timed up-and-go test with cognitive task (TUG-dual) is recommended for assessing such problem but focusing only on total TUG time may mask the extent of cognitive tasks affecting each TUG component. This study investigates the effect of age and educational level on dual task performance during each component of TUG in patients with stroke. This cross-sectional study evaluated 25 patients with stroke and 25 healthy persons when performing $7 \mathrm{~m}$ TUG with and without counting backward by three. Total time to perform TUG, movement parameters, and rates of counting correct answers were measured. Four components of $7 \mathrm{~m}$ TUG (sit-to-stand, walk, turn, and turn-to-sit) were classified using portable accelerometers. The duration of TUG-dual increased in both groups but the amount of increase was larger in patients $(p<0.001)$. The rate of counting correct answer was affected by age $(p=0.004)$, but not educational levels $(p=0.267)$. In patients, the decreased rate of counting was found across all age ranges (35-54, 55-64, and 65-78 years), but in healthy persons, it was found only in the oldest age range. Cognitive tasks led to longer time during walk, turn, and turn to sit in patients. Changes in movement parameters including decreased peak angular velocity during turn and turn-to-sit, decreased stride length, stride velocity, and increased single leg stance time during walking suggested adaptation patterns in patients with stroke.
\end{abstract}

KEYWORDS: cerebrovascular accident, gait, turning, balance

\section{INTRODUCTION}

Everyday activities contain simultaneous performance of motor and cognitive tasks such as talking while walking. Patients who have had a stroke have problems dealing with cognitive-motor dual tasking. Reduction in motor performance during walking, including decreased walking speed ${ }^{1}$, increased stride length and stride time ${ }^{2}$, increased double limb support duration ${ }^{3}$, and stop walking ${ }^{4}$ is evident when patients are challenged by cognitive tasks during walking. The effect of cognitive task on gait performance is more pronounced during the more attention demanding task, as shown by decreased gait speed, decreased stride time before turning and increased time to turn ${ }^{5}$ as well as decreased number of correct cognitive response ${ }^{6}$. $\mathrm{Age}^{7}$ and education level ${ }^{8}$ are the important factors for determining cognitive function. However, the effect of adding cognitive task on gait performance in persons with stroke who have different ages and level of educational attainment have not been revealed.

The timed up-and-go (TUG) test is the clinical test recommended for measuring basic mobility skills ${ }^{9}$, especially walk to turn, in patients with stroke $^{10}$. Cognitive task such as number subtraction has been introduced during the TUG to assess the cognitive-motor interference. The TUG test begins with rising from a chair, walking for $3 \mathrm{~m}$ at a comfortable pace, turning, walking back to the starting point and sitting down on the chair. This test is quantified by timing the duration from the start command until the buttocks touch the chair ${ }^{9}$. However, the total time for completing a TUG test appears to be an inadequate measurement of fall risk $^{10}$ and fails to detect change after three months post-stroke ${ }^{11}$. Focusing only on total time prevents 
a specific impairment to be determined within the TUG test. Prolonged TUG time after stroke may be due to deficits at any event of movement that requires balance control, such as during postural transition ${ }^{12}$ and turning ${ }^{13}$. In training of a serial complex task, the trainer may need to break a complex task into several subcomponent and train each component separately before training the whole task $^{14}$. Breaking a task into subcomponent may help facilitate motor learning in persons with stroke who have impairment at the central nervous system level. Hence information regarding the movement parameters in each component of the TUG such as stride time, stride length during straight walking component, peak angular velocity of trunk movement during turning component will be useful for training those with stroke in clinic.

Performance on the components of the TUG can be investigated using various equipment such as a multimemory stopwatch and inertial sensor-based assessment of movements. Wall et al has developed the Expanded Timed Get Up and Go test which uses the longer walkway $(10 \mathrm{~m})$ to allow more accuracy in assessing the walking component in TUG ${ }^{15}$. More recently (2010), Salarian et al demonstrated that 7$\mathrm{m}$ walkway was reliable and valid for assessment of TUG using inertial sensors as it could provide enough steps for analysis of movement parameters, such as stride length and stride time in patient with Parkinson's disease ${ }^{16}$.With similar instrument, we therefore employed the 7-m walking distance in the present study aiming to investigate the effects of adding cognitive tasks to component of $7 \mathrm{~m}$ TUG in patients with stroke compared to healthy persons. We hypothesized that the effect of adding cognitive tasks on TUG performance would be different among stroke patients with different ages and educational attainment and adding cognitive task on TUG would affect components of 7 m TUG unequally based on the attention requirement of each TUG component.

\section{MATERIALS AND METHODS}

\section{Setting}

This cross-sectional study was carried out in patients with subacute stroke and healthy persons at Prasart Neurological Institute (PNI), Bangkok, Thailand. Data were collected at the rehabilitation unit of PNI and analysed at Faculty of Physical Therapy, Srinakharinwirot University and Faculty of Science, Mahidol University, Thailand.

\section{Participants}

Patients with a medical diagnosis of stroke (code I60-I62: non-traumatic intracranial haemorrhage and I63: cerebral infarctions) were invited to participate in the study. Those included in the study were between 38 and 78 years old with stroke and able to walk independently for $14 \mathrm{~m}$ without using a walking aid. Patients were excluded from the study if they had heart diseases; multiple brain lesions; cerebral aneurysm, brainstem or cerebellar lesions; neurological or musculoskeletal disorders other than stroke that was sufficient to disturb balance; and comprehension problems, defined as having a MiniMental State Examination (MMSE) Thai version score of less than $24^{17}$. Healthy participants, ageand height-matched, were recruited from volunteers who lived or worked close to PNI. Sample size was calculated using our pilot data based on duration of turning component of the TUG and the TUG with cognitive task as it is related to turning capacity following stroke. Fifty participants, 25 in each group, were obtained to yield the power of 0.95 at $p<0.05$. The actual number of the recruited participant was equal to the calculated sample size. All participants were provided informed consent approved by the Institutional Review Board of PNI.

\section{Tasks and procedure}

Baseline information including age, height, weight, and educational level was collected in all participants using the questionnaire. Motor and balance performance of participants with stroke were determined using the Fugl-Meyer Assessment motor subscale (FM-motor) ${ }^{18}$ and the Berg Balance Scale (BBS) ${ }^{19}$. The FM-motor includes examination of upper (FM-UE) and lower extremities (FM-LE) function with a maximum score of 66 and 34 for the FMUE and the FM-LE, respectively ${ }^{18}$. The BBS contains 14 items of functional balance task. Its total score of 56 can be rated on a 4-point ordinal scale.

The performance of three main tasks, cognitive task when seated (cognitive-single), timed up-andgo (TUG) with and without cognitive task were examined in all participants. Each participant started with cognitive-single to ensure that he/she can perform the cognitive task correctly, followed by a random order of either 7 m TUG without cognitive task (7 m TUG-single) or with cognitive task (7 m TUG-dual). Each subject was required to perform each task only once during data collection with five minutes of resting between the tasks. Trials were video-audio recorded for further analysis of 
cognitive performance. The cognitive task chosen in this study was counting numbers backwards by three, starting with a number randomized from 100 to 70 , in order to reduce the learning effect ${ }^{5}$. Prior to data collection, all tasks were demonstrated and practised until the participants became familiar with the tasks.

During the cognitive-single, participants counted numbers backwards by three for $30 \mathrm{~s}$ when sitting on a chair with their back supported. For $7 \mathrm{~m}$ TUG-single, participants performed series of activities in $7 \mathrm{~m}$ TUG, including standing from a chair, straight walking for $7 \mathrm{~m}$, turning, walking back and turning to sit on the chair. Participants started the performance after a 'go' command. They were instructed to stand up without using hand support and walk barefoot at a comfortable pace. The total duration of $7 \mathrm{~m}$ TUG was calculated when participants moved their back away from the chair until they sat down and their back touched the chair again. During the $7 \mathrm{~m}$ TUG-dual, participants were asked to count numbers backward by three for $5 \mathrm{~s}$ before starting the $7 \mathrm{~m}$ TUG task at the go command. They were then instructed to perform the $7 \mathrm{~m}$ TUG task simultaneously with the counting task until the $7 \mathrm{~m}$ TUG task was completed, without prioritizing one over the other. Hence the time for performing cognitive task varied between participants depending on the time used to complete the $7 \mathrm{~m}$ TUG task dual task. To ensure that varying time for counting did not affect our main result, 10 participants (2 patients with stroke and 7 healthy persons) were assessed in the pilot study. In the pilot study, each participant was asked to count backward in sitting position six times. Each time the duration was varied from $15,30,45,50,75,90 \mathrm{~s}$ (the longest possible duration for walking in persons with stroke) and the sequence of counting was randomly assigned. No relationship ( $r=-0.178, p=0.175$ ) between counting duration and number of correct answers when counting was observed.

\section{Data collection and analysis}

The instrumented timed-up and go (iTUG), APDM Mobility Lab system (APDM, Inc., Portland, USA), a set of portable inertial sensors and software were used to classify the components of $7 \mathrm{~m}$ TUG. The iTUG is reliable and valid to measure TUG performance in several group of patients with neurological diseases ${ }^{16,20}$. The iTUG has excellent testretest reliability (intraclass correlation coefficients range 0.43-0.99) and duration components of the

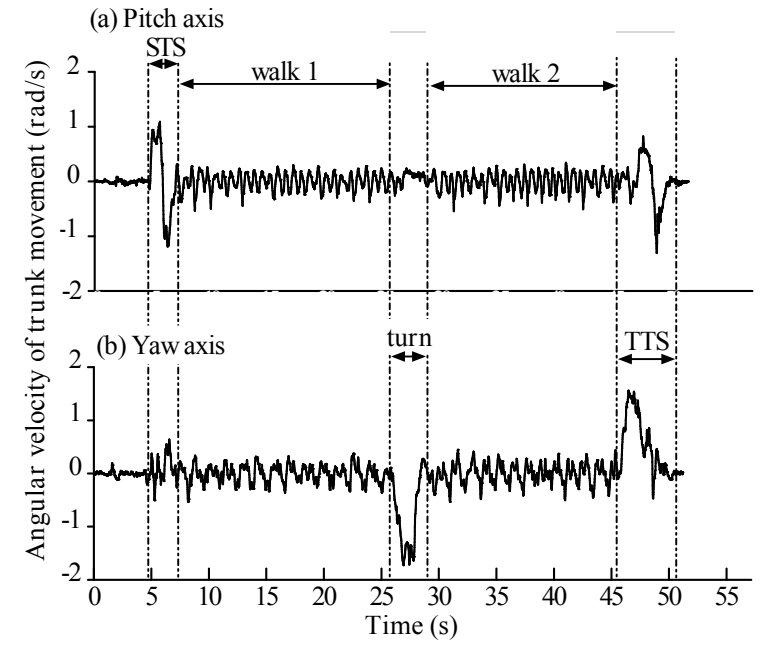

Fig. 1 Raw signals derived from inertial sensor on the trunk. Peak angular velocity of trunk movement corresponds to postural transition determines components of the $7 \mathrm{~m}$ TUG task in rad/s. (a) The first peak on pitch angular velocity signal corresponds to sit-to-stand (STS). (b) Yaw angular velocity signal, the first peak corresponds to the $180^{\circ}$ turn (turn) and the second peak corresponds to the turn-to-sit transition (TTS). The positive value of pitch and yaw signals represents trunk forward movement in sagittal plane and trunk rotation to the right-hand side of the participant, respectively.

TUG were valid to differentiate performance between patients with stroke and healthy persons ${ }^{20}$. Six portable 3 dimensional inertial sensors were placed at mid-thoracic, 5th lumbar vertebra, bilateral wrists and ankles ${ }^{21}$ A gyroscope $\left( \pm 400^{\circ} / \mathrm{s}\right.$ range) and accelerometer ( $\pm 5 \mathrm{~g}$ range) captured angular velocity and acceleration at the sampling rate of $200 \mathrm{~Hz}^{22}$. An example of $7 \mathrm{~m}$ TUG component identification is shown in Fig. 1. Four components of $7 \mathrm{~m}$ TUG, including sit-to-stand (STS), total walk (walk1+walk2), $180^{\circ}$ turn (turn) and turn-to-sit (TTS) were identified by trunk velocity and acceleration using APDM iTUG software. The duration of each $7 \mathrm{~m}$ TUG component and movement parameters including peak angular velocity of trunk movement during each component, and stride length, stride velocity, and single leg stance time (SLS) during walk and step time and number of step during turn were also calculated by APDM iTUG software $^{16}$. To determine cognitive performance, the rate of counting correct answers (number of correct answer(s)) was analysed by dividing the number of correct answers by $30 \mathrm{~s}$ during sitting or dividing the number of correct answers by total 
$7 \mathrm{~m}$ TUG duration of the same trial during walking. The numbers of participants who stopped counting during each $7 \mathrm{~m}$ TUG component and number of correct answers were determined from the recorded video by two raters. Prior to data collection, raters have been trained to use the equipment reliably and accurately.

An independent $t$-test was used for comparing age, height, and weight between patients and healthy persons. Two-way ANOVA was used to examine the effect of task (single and dual tasks) and group (patients with stroke and healthy persons) on total duration of the TUG, duration and movement parameters of each component of the $7 \mathrm{~m}$ TUG. The comparison of spatiotemporal parameters between groups was matched for patient's affected leg versus healthy person's non-dominant leg, and patient's unaffected leg versus healthy person's dominant leg. Statistical significances were set at $p<0.05$. Age was classified into 3 categories; $35-54,55-64$, and $65-78$ years according to agespecific incidence rates for first-ever stroke ${ }^{23}$ and the levels of educational attainment (degree, high school, and elementary) were classified according to classification of education in Thailand. The effect of age and educational on the rate of counting correct answer during total $7 \mathrm{~m}$ TUG were analysed using MANOVA. Tukey post hoc tests were used to identify differences among the three age ranges. Significant group and interaction effects were set at $p<0.05$.

\section{RESULTS}

\section{Participants}

Table 1 shows the demographics and clinical characteristics of participants; 25 patients post-stroke and 25 age- and height-matched healthy persons. The average age, height, weight, and score of MMSE in patients and healthy participants were not statistically significant difference (Table 1).

\section{Total $7 \mathrm{~m}$ TUG duration and rate of counting correct answer}

During $7 \mathrm{~m}$ TUG-single, mean total duration was $45 \pm 14 \mathrm{~s}$ in patients and $20.4 \pm 4.2 \mathrm{~s}$ in healthy persons; while, during $7 \mathrm{~m}$ TUG-dual, mean total duration was $61 \pm 20 \mathrm{~s}$ in patients and $26.8 \pm 6.7 \mathrm{~s}$ in healthy persons (Fig. 2). Patients showed significant longer total duration than healthy persons during both $7 \mathrm{~m}$ TUG-single and $7 \mathrm{~m}$ TUG-dual $\left(\mathrm{F}_{(1,8)}=47.05, p<0.001\right)$. In addition, both groups of subjects showed increased total duration under $7 \mathrm{~m}$ TUG-dual when compared to $7 \mathrm{~m}$ TUG-single
Table 1 Demographic and clinical characteristics of participants!

\begin{tabular}{|c|c|c|c|c|}
\hline \multirow[t]{2}{*}{ Variable } & \multicolumn{2}{|c|}{ Stroke $(n=25)$} & \multicolumn{2}{|c|}{ Healthy $(n=25)$} \\
\hline & Mean \pm SD & Range & Mean \pm SD & Range \\
\hline Height $(\mathrm{cm})$ & $164 \pm 11$ & $145-189$ & $164 \pm 9$ & $150-180$ \\
\hline Weight (kg) & $65 \pm 11$ & $45-85$ & $67 \pm 10$ & $52-90$ \\
\hline MMSE $(/ 30)$ & $29.5 \pm 1.3$ & $26-30$ & $29.0 \pm 1.5$ & $27-30$ \\
\hline Gender & $\mathrm{M}=14$ & $\mathrm{~F}=11$ & $\mathrm{M}=14$ & $\mathrm{~F}=11$ \\
\hline Age (y) & $57 \pm 12$ & $27-78$ & $55 \pm 12$ & $26-76$ \\
\hline Age 35-54 (y) & \multicolumn{2}{|c|}{$45.8 \pm 5.6(11)$} & \multicolumn{2}{|c|}{$45.9 \pm 5.2(11)$} \\
\hline Age 55-64 (y) & \multirow{2}{*}{\multicolumn{2}{|c|}{$59.1 \pm 2.8(8)$}} & \multirow{2}{*}{\multicolumn{2}{|c|}{$\begin{array}{l}60.6 \pm 2.4(8) \\
70.2 \pm 5.8(6)\end{array}$}} \\
\hline Age 65-78 (y) & & & & \\
\hline \multicolumn{5}{|l|}{ Education } \\
\hline - Elementary & \multicolumn{2}{|l|}{2} & \multicolumn{2}{|l|}{5} \\
\hline - High School & \multirow{2}{*}{\multicolumn{2}{|c|}{14}} & \multicolumn{2}{|l|}{12} \\
\hline - Degree & & & \multirow{2}{*}{\multicolumn{2}{|c|}{8}} \\
\hline Ischemic type & \multicolumn{2}{|c|}{22} & & \\
\hline Hemorrhage type & \multicolumn{2}{|l|}{3} & \multicolumn{2}{|l|}{-} \\
\hline Hemiplegic side & $\mathrm{R}=12$ & $\mathrm{~L}=13$ & \multicolumn{2}{|l|}{ - } \\
\hline TSS (day) & $23 \pm 35$ & $2-120$ & \multicolumn{2}{|l|}{ - } \\
\hline FM-motor $(/ 100)$ & $75 \pm 21$ & $28-98$ & \multicolumn{2}{|l|}{ - } \\
\hline FM-UE $(/ 66)$ & $47 \pm 19$ & $9-65$ & \multicolumn{2}{|l|}{ - } \\
\hline FM-LE (/54) & $28 \pm 4$ & $19-33$ & \multirow{2}{*}{\multicolumn{2}{|c|}{-}} \\
\hline BBS $(/ 56)$ & $40 \pm 15$ & $8-55$ & & \\
\hline
\end{tabular}

${ }^{\dagger}$ MMSE $=$ Mini-mental state examination; TSS=Time since stroke, FM-motor=Fugl-Meyer assessment motor subscale; FM-UE=FM-motor assessment of the upper extremity; FM-LE=FM-motor assessment of the lower extremity; BBS=Berg balance scale. The comparison of age, height, and weight between patients and healthy persons was done using an independent t-test.

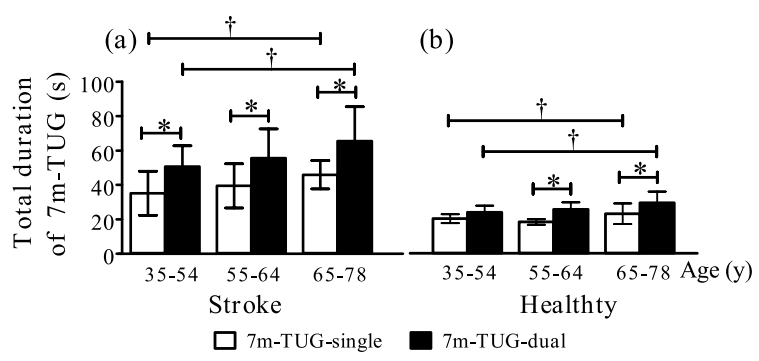

Fig. 2 Total duration of the modified version of expended timed get up and go test (7 m TUG). Mean total duration ( \pm SD) of the $7 \mathrm{~m}$ TUG without (7 m TUG-single) and with cognitive task (7 m TUG-dual) in (a) patients with stroke and (b) healthy persons. * Significant difference between $7 \mathrm{~m}$ TUG-single and $7 \mathrm{~m}$ TUG-dual; $\uparrow$ significant task-group interaction.

$\left(\mathrm{F}_{(1,8)}=22.85, p=0.001\right)$, but the effect of adding cognitive tasks on total $7 \mathrm{~m}$ TUG duration was more pronounced in patients than in healthy persons, as shown by significant task-group interaction $\left(\mathrm{F}_{(1,8)}=\right.$ $1.37, p=0.045)$.

During cognitive-single, the rate of counting correct answers between patients $(0.31 \pm 0.08$ number/s) and healthy persons $(0.33 \pm 0.11$ number/s $)$ 


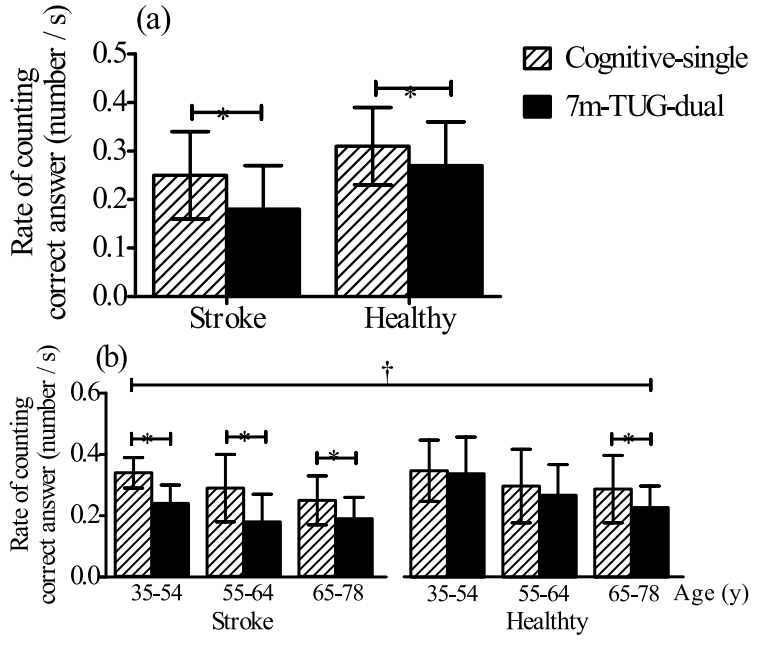

Fig. 3 Rate of counting correct answer. (a) Effect of task and group, mean $( \pm S D)$ rate of counting correct answer during cognitive-single and 7 m TUG-dual (tasks) in patients with stroke and healthy persons (groups). (b) Effect of age, mean $( \pm S D)$ rate of counting correct answer reported in different age ranges (35-54, 55-64, and 65-78 years). * Significant difference between tasks; $\dagger$ significant age-group interaction.

was not significantly different $\left(\mathrm{F}_{(1,8)}=0.16, p=\right.$ $0.690)$. The rate of counting correct answer reduced in both groups (Fig. 3a) at the similar rate during 7 m TUG-dual, as shown by no significant taskgroup interaction $\left(\mathrm{F}_{(1,8)}=8.04, p=0.026\right)$. However, some patients stopped counting during $7 \mathrm{~m}$ TUG-dual, specifically during walking (2 patients), turning and turning to sit (8 patients).

\section{The effect of age and educational attainmen}

Ages of participants did not significantly affect $7 \mathrm{~m}$ TUG duration $\left(\mathrm{F}_{(2,8)}=2.32, p=0.107\right)$ but significantly affected the rate of counting correct answer $\left(\mathrm{F}_{(2,8)}=6.96, p=0.004\right)$; however, educational attainment levels of participants did not significantly affect the rate of counting correct answer $\left(\mathrm{F}_{(2,8)}=\right.$ 1.52, $p=0.107)$. During $7 \mathrm{~m}$ TUG-single and $7 \mathrm{~m}$ TUG-dual, older patients and healthy persons (age range, 65-78 years) showed slower rate of counting correct answer than younger persons (age range, 36-44 years) (post hoc ANOVA; $p$ :0.001). During $7 \mathrm{~m}$ TUG-single, in patient group, the decreased rate of counting correct answer was found across all age ranges (35-54, 55-64, and 65-78 years), but in healthy group, the change was found only in the oldest age range $65-78$ years (Fig. 3b). The effect of age was more pronounced in patients than

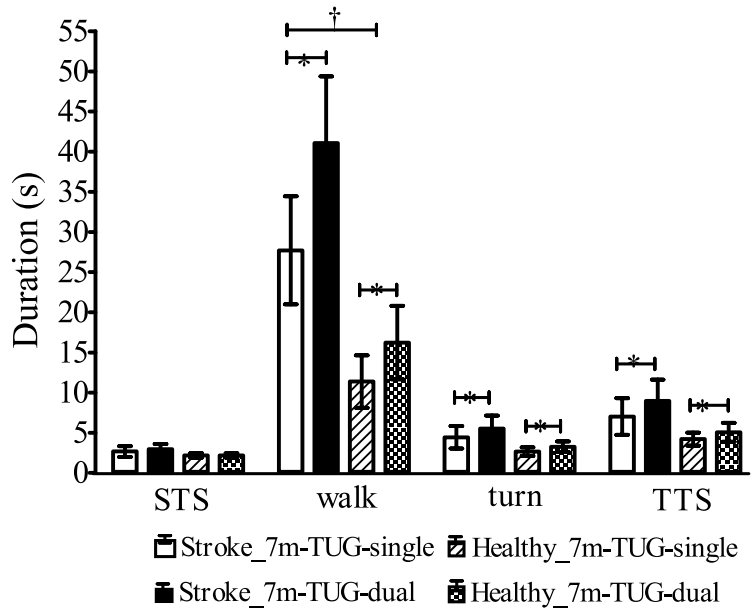

Fig. 4 Mean duration of TUG components during $7 \mathrm{~m}$ TUG-single and $7 \mathrm{~m}$ TUG-dual in patients with stroke and healthy persons. * Significant difference between TUGsingle and TUG-dual; $\uparrow$ significant task-group interaction; STS $=$ sit-to-stand, walk $=$ total walk, turn $=180^{\circ}$ turn, and TTS $=$ turn-to-sit.

in healthy persons, as shown by significant agegroup interaction on rate of counting correct answer $\left(\mathrm{F}_{(5,8)}=6.98, p=0.009\right)$.

\section{$7 \mathrm{~m}$ TUG components analysis}

Analysis of $7 \mathrm{~m}$ TUG components revealed that patients had a significantly longer duration of all components; STS, walk, turn and TTS, than healthy persons (Fig. 4) $\left(\mathrm{F}_{(1,96)}=78.40-113.09\right.$, $p<0.001)$. During $7 \mathrm{~m}$ TUG-dual, both groups demonstrated longer duration in all $7 \mathrm{~m}$ TUG components $\left(\mathrm{F}_{(1,96)}=1.96-22.88, p<0.001\right)$, except STS $\left(\mathrm{F}_{(1,96)}=1.73, p=0.190\right)$ (Fig. 4). However, the increase in duration of walk component of the $7 \mathrm{~m}$ TUG was more pronounced in patients than in healthy persons, as shown by significant interaction effect (Fig. 4). $\left(\mathrm{F}_{(1,84)}=4.07, p=0.004\right)$.

The effect of adding cognitive task on movement parameters within each the component of the TUG was evident in both persons with stroke and healthy groups but the larger effects were found in patients with stroke (Fig. 5 and Fig. 6). The effect of adding cognitive task on peak angular velocity of trunk movement was found only during turn and TTS in both groups, where patients demonstrated much lower peak angular velocity of trunk movement than healthy persons (Fig. 5). For the analysis of walk components of the TUG (Fig. 6), patients demonstrated lower stride length, lower stride velocity and shorter single leg stance time 


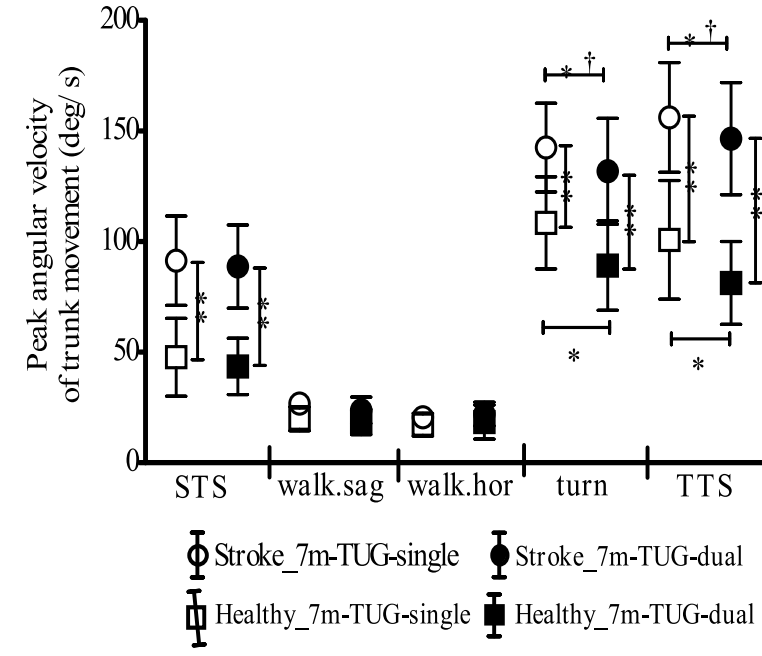

Fig. 5 Mean and SD of peak angular velocity of trunk movement under $7 \mathrm{~m}$ TUG-single and $7 \mathrm{~m}$ TUG-dual. STS $=$ sit-to-stand component, walk.sag $=$ walk component (sagittal plane), walk.hor $=$ walk component (horizontal plane), turn $=180^{\circ}$ turn component, and TTS =turn-to-sit component. * significant difference between TUG-single and TUG-dual; ** significant difference between groups; $\uparrow$ significant task-group interaction.

than the healthy persons. Adding cognitive task led to significantly lower stride length of the unaffected limb (Fig. 6a), significant decreased stride velocity of both affected and unaffected limbs (Fig. 6b), decreased single leg stance duration of affected leg and increased single leg stance duration of unaffected leg (Fig. 6c). Healthy persons were also more affected by TUG-dual with fewer changes (i.e., decreased stride velocity of both legs) than patients (Fig. 6b).

During TUG-single, the average number of step during turn was 6.88 steps with an average step time of $0.78 \mathrm{~s} / \mathrm{step}$ in patients and 5.52 steps with $0.59 \mathrm{~s} / \mathrm{step}$ in healthy persons. Under TUGdual, the average number of steps during turn was 7.20 steps with $0.88 \mathrm{~s} / \mathrm{step}$ in patients and 5.65 steps with $0.69 \mathrm{~s} /$ step in healthy persons. Patients demonstrated significant higher number of steps $\left(\mathrm{F}_{(1,48)}=9.82, p<0.01\right)$ and longer step time $\left(\mathrm{F}_{(1,48)}=27.28, p<0.01\right)$ when compared to healthy persons during TUG-single and TUG-dual. Cognitive dual task affected both groups, as can be seen by significantly longer step time during turn $\left(\mathrm{F}_{(1,48)}=19.96, p<0.01\right)$, while number of steps remained unchanged $\left(\mathrm{F}_{(1,48)}=0.80, p=0.37\right)$.
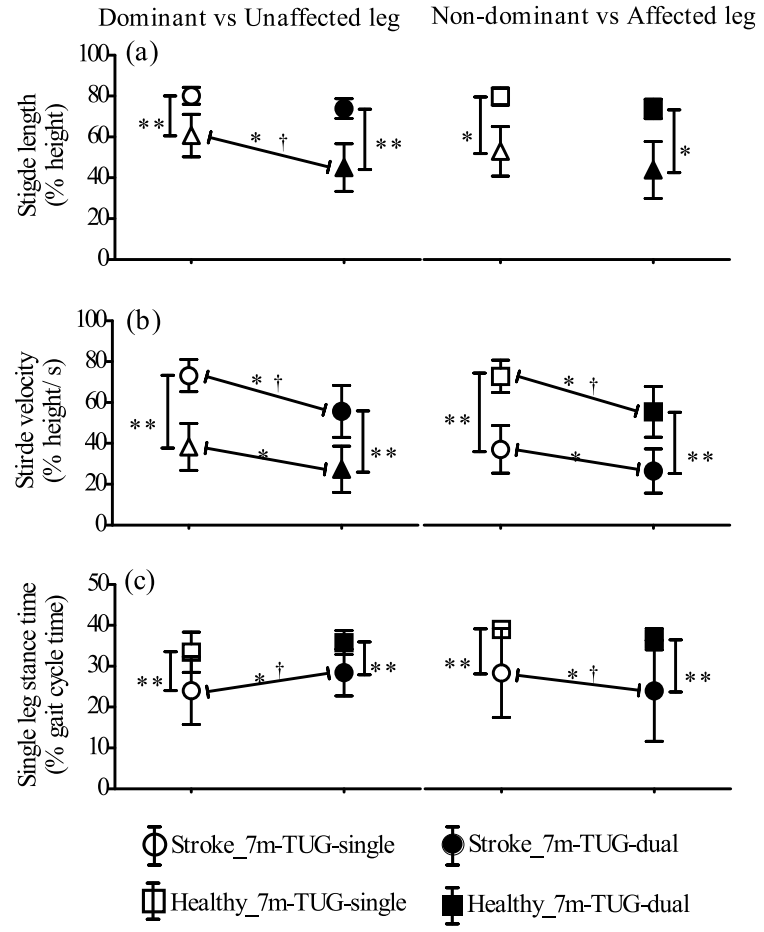

Fig. 6 Mean and SD of spatiotemporal parameters during walk component; (a) stride length, (b) stride velocity and (c) single leg stance duration under TUG-single and TUGdual in patients with stroke and healthy persons. The left panel shows comparison between patients' unaffected leg and healthy's dominant leg and the right panel shows comparison between patients' affected leg and healthy's non-dominant leg. * Significant difference between TUGsingle and TUG-dual; ** significant difference between patients and healthy persons; $\uparrow$ significant task-group interaction.

\section{DISCUSSION}

This study aimed to clarify the effect of cognitive dual task on the components of $7 \mathrm{~m}$ timed up-and-go Test in patients with stroke. Results supported the hypothesis that there is a relationship between the degree of cognitive interference and components of TUG. The finding that patients with stroke and healthy persons increased time to complete $7 \mathrm{~m}$ TUG-dual as compared to $7 \mathrm{~m}$ TUG-single was in line with the previous literature, where elderly persons ${ }^{24}$ and individuals with stroke ${ }^{6}$ took longer to complete the cognitive TUG test as well as decrease rate of counting correct answer. A decline in performance of either cognitive or motor tasks, or both, under dual-task conditions has been described as cognitive-motor interference (CMI) ${ }^{25}$. The interference between central processing for cognitive and 
motor task occurs when tasks that share a common processing are concurrently performed. The amount of interference is related to the attention requirement of tasks, such that attention requirement that exceeds the limited central processing resources results in declined performance in either or both tasks, as compared to the performance of each task separately $^{26}$.

Patterns of CMI are classified into 9 categories; no change in task performance (no interference), decreased motor performance only (cognitive-related motor interference), stable motor performance with decreased cognitive performance (motor-related cognitive interference), declined performance of both motor and cognitive tasks relative to each other (mutual interference), increased performance of motor (motor facilitation) or cognitive performance (cognitive facilitation), increased performance of one task with declined performance of the other task (cognitive-priority trade-off or motor-priority trade-off), and increased performance of both tasks (mutual facilitation) ${ }^{25}$. In our study, two CMI patterns were found in healthy group. Healthy young persons demonstrated decreased $7 \mathrm{~m}$ TUG performance without a significant change in cognitive performance, suggesting cognitive-related motor interference. In contrast, healthy older adults showed decreased in both TUG and cognitive performances. This is classified as mutual interference pattern. A possible reason for CMI difference between young and older healthy persons is due to age-related working memory effectiveness. Counting backward task used in the present study is a type of working memory task that requires central processing to continue subtracting number.

Our finding of decreased performance of working memory task in healthy older persons corresponds with previous reported age-related percent of correct subtraction ${ }^{27}$. Age-related decreasing in performance of working memory task may be from reduced cognitive resources available for processing $^{28}$ and slowing of computational processes ${ }^{29}$. In addition, it has been shown that the ability to control balance reduced with age, thus attention requirement for maintaining balance is higher for older adults than younger subjects ${ }^{26}$. When performing multiple tasks simultaneously, the older adult may not have the capacity to perform both tasks, resulting in deterioration of both tasks ${ }^{26}$. A possible reason for no effect of education on cognitive task is that the counting backward task is a simple math task for all levels of educational attainments of our participants.
A decrease of both $7 \mathrm{~m}$ TUG and cognitive performance in patients with stroke, although with a larger deterioration than healthy, indicated mutual interference. Result on mutual interference during $7 \mathrm{~m}$ TUG-dual in patients with stroke corresponded with previous studies that measured performance when standing ${ }^{30}$ or walking ${ }^{31}$. Cognitive impairment might not be a reason for such decreased cognitive performance in patients as they had normal cognitive levels and their MMSE scores were not different from those of healthy persons under cognitive-single. The possible reason for mutual interference in the patients may be due to limited postural reserve which could lead to high attention requirements for performing postural transition activities $^{32}$. In the present study, patients with stroke had lower mobility and balance performance than healthy persons, thus low motor and balance capability could lead to higher attention requirement for controlling postural stability during $7 \mathrm{~m}$ TUG-dual. As a result, attention requirements may exceed capacity limits, leading to a larger decrease in both cognitive and motor performance in patients.

Our results also demonstrated that patients spent longer time during the walk, turn and TTS, but not the STS component. Some of patients stop counting during walk, turn, and TTS, but not STS and walk. In addition, only the increase in duration of walk component was more pronounced in patients than healthy persons. These results indicated that attention allocation for each TUG component was not equal; the largest attention demand occurred during walking, and the lowest was seen during STS. This finding could be partly explained by the requirement of large attention demand for persons with stroke to control lower extremity during walk. In the present study, all patients could walk without using gait aid; however, they still had some degree of lower extremity motor impairment (mean score of FM-LE $=28 / 54$ ) and balance impairment (mean score of BBS $=40.35 / 56$ ). These impairments may cause higher requirement for cortical control of walking ${ }^{33}$. Turning requires the coordination of eyes, head, limbs and trunk to provide anticipatory adjustments before changing the CoM position during sitting down ${ }^{34}$. However, short period of this activity may lead to less attention demand for carrying out dual-task compared to a long distance of walking component of $7 \mathrm{~m}$ TUG task. In contrast, sit to stand involves the control of trunk and limb movements to generate forward and upwards momentum of the $\mathrm{CoM}^{35}$; thus it may require less cortical control. 
Changes in movement parameters suggested a compensatory strategy to maintain gait and balance when the attention was allocated for both cognitive task and TUG task. The decreased peak angular velocity of trunk movement during turn and TTS indicated the modulation of trunk rotation and step time during these TUG components when participants were distracted with cognitive tasks. Although both healthy older persons and patients showed decreased trunk turning velocity, the amount of decrease was larger in the patients. These characteristics were reported as turning difficulty in patients with chronic stroke ${ }^{5,34}$. On the other hand, this strategy may be accounted for by a cautious strategy or turning with safety ${ }^{36}$. We also observed the compensatory strategy during walk component of TUG-dual, but those strategies were not the same between healthy persons and patients. Healthy older persons adjusted speed of walking by decreasing stride velocity, leading to longer duration of walk component in TUG-dual. These findings were in line with a previous study reporting similar characteristics of gait modulation in elderly individuals who were challenged with dual tasks during walking ${ }^{37}$. In contrast, during TUGdual, patients with stroke decreased stride velocity as well as demonstrated excessive asymmetrical gait pattern, as shown by decreased single leg stance time of affected leg and increased single leg stance time of unaffected leg. This compensation reflected inability to maintain stability of the moving CoM over the effected stance leg in patients with stroke when challenging with dual task ${ }^{38}$, which could explain the need to decrease stride velocity and stride length in order to gain stability during walk component of TUG-dual.

Findings of this study were based on the use of the $7 \mathrm{~m}$ TUG where the walkway was $7 \mathrm{~m}$ instead of $3 \mathrm{~m}$, thus the results from this study may differ when the original TUG test was used. The cognitive task examined in this study is the arithmetic task of counting backward by three. It is not known whether other types of cognitive task would affect TUG components in a similar way as the arithmetic task does. This question could be explored further in the future study. In addition, our study was carried out in high functioning persons with stroke who could walk without using a walking aid. The clinician should apply these findings specifically in patients with high mobility function.

Information from this study provides clinical implications for stoke rehabilitation. Although mutual interference during dual task walking found in both patients and healthy persons, the deterioration in walking performance was more pronounced in patients. Due to mutual interference pattern, training both motor and cognitive performances under dual tasking activity should be routinely practised in patients with stroke, even in those who can walk without using assistive devices. Performance assessment and training during dual task should focus on both walking and cognitive task. However, the training could be simplified for the beginners, for example, using treadmill during dual task training for improving automatic of walking (less attention requirement) and gaining community walking ability in patients with stroke.

In conclusion, the present study demonstrated that the effect of adding cognitive task of counting backward on TUG performance was varied among stroke patients with different age ranges, but not with different levels of educational attainment. The cognitive task affected components of TUG differently, depending on the cognitive requirement of the task in that component. Declining motor performance during TUG-dual, and the changes of acceleration-based movement parameters especially during walk, turn and TTS components found in patients with stroke, may help guide assessment and interventions directly towards components of the TUG that are more problematic.

Acknowledgements: This work was supported by the under Grant from the Thailand Research Fund, Office of the Higher Education Commission (RSA5580002).

\section{REFERENCES}

1. Patel P, Lamar M, Bhatt T (2014) Effect of type of cognitive task and walking speed on cognitive-motor interference during dual-task walking. Neuroscience 260, 140-8.

2. Plummer-D'Amato P, Altmann LJ, Saracino D, Fox E, Behrman AL, Marsiske M (2008) Interactions between cognitive tasks and gait after stroke: A dual task study. Gait Posture 27, 683-8.

3. Plummer-D'Amato P, Altmann LJ, Behrman AL, Marsiske M (2010) Interference between cognition, double-limb support, and swing during gait in community-dwelling individuals poststroke. Neurorehabil Neural Repair 24, 542-9.

4. Weerdesteyn V, van Swigchem R, van Duijnhoven HJ, Geurts AC (2007) Why stroke patients stop walking when talking. J Am Geriatr Soc 55, 1691.

5. Hollands KL, Agnihotri D, Tyson SF (2014) Effects of dual task on turning ability in stroke survivors and older adults. Gait Posture 40, 564-9. 
6. Manaf H, Justine M, Goh HT (2015) Effects of attentional loadings on gait performance before turning in stroke survivors. $P M \& R$ 7, 1159-66.

7. Brown SC, Park DC (2003) Theoretical models of cognitive aging and implications for translational research in medicine. Gerontologist 43, 57-67.

8. Hatch SL, Feinstein L, Link BG, Wadsworth MEJ, Richards M (2007) The continuing benefits of education: adult education and midlife cognitive ability in the British 1946 birth cohort. J Gerontol B Psychol Sci Soc Sci 62, S404-14.

9. Flansbjer UB, Holmback AM, Downham D, Patten C, Lexell J (2005) Reliability of gait performance tests in men and women with hemiparesis after stroke. $J$ Rehabil Med 37, 75-82.

10. Hafsteinsdottir TB, Rensink M, \& Schuurmans M (2014) Clinimetric properties of the timed up and go test for patients with stroke: a systematic review. Top Stroke Rehabil 21, 197-210.

11. Persson CU, Danielsson A, Sunnerhagen KS, GrimbyEkman A, Hansson PO (2014) Timed up \& go as a measure for longitudinal change in mobility after stroke - postural stroke study in Gothenburg (POSTGOT). J Neuroeng Rehabil 11, 83.

12. Lomaglio MJ, Eng JJ (2005) Muscle strength and weight-bearing symmetry relate to sit-to-stand performance in individuals with stroke. Gait Posture 22, 126-31.

13. Hollands KL, Hollands MA, Zietz D, Wing AM, Wright C, van Vliet P (2010) Kinematics of turning $180^{\circ}$ during the timed up and go in stroke survivors with and without falls history. Neurorehabil Neural Repair 24, 358-67.

14. Fontana FE, Mazzardo O, Furtado O, Jr., Gallagher JD (2009) Whole and part practice: a meta-analysis. Percept Mot Skills 109, 517-30.

15. Wall JC, Bell C, Campbell S, Davis J (2000) The timed get-up-and-go test revisited: measurement of the component tasks. J Rehabil Res Dev 37, 109-13.

16. Salarian A, Horak FB, Zampieri C, Carlson-Kuhta P, Nutt JG, Aminian K (2010) iTUG, a sensitive and reliable measure of mobility. IEEE Trans Neural Syst Rehabil Eng 18, 303-10.

17. Tombaugh TN, McIntyre NJ (1992) The mini-mental state examination: a comprehensive review. $J \mathrm{Am}$ Geriatr Soc 40, 922-35.

18. Sanford J, Moreland J, Swanson LR, Stratford PW, Gowland C (1993) Reliability of the Fugl-Meyer assessment for testing motor performance in patients following stroke. Phys Ther 73, 447-54.

19. Berg KO, Maki BE, Williams JI, Holliday PJ, WoodDauphinee SL (1992) Clinical and laboratory measures of postural balance in an elderly population. Arch Phys Med Rehabil 73, 1073-80.

20. Wuest S, Masse F, Aminian K, Gonzenbach R, de Bruin ED (2016) Reliability and validity of the inertial sensor-based timed 'up and go' test in individuals affected by stroke. J Rehabil Res Dev 53, 599-610.

21. Najafi B, Aminian K, Paraschiv-Ionescu A, Loew F, Bula CJ, Robert P (2003) Ambulatory system for human motion analysis using a kinematic sensor: monitoring of daily physical activity in the elderly. IEEE Trans Biomed Eng 50, 711-23.

22. Salarian A, Russmann H, Vingerhoets FJ, Dehollain C, Blanc Y, Burkhard PR, Aminian K (2004) Gait assessment in Parkinson's disease: toward an ambulatory system for long-term monitoring. IEEE Trans Biomed Eng 51, 1434-43.

23. Kissela BM, Khoury JC, Alwell K, Moomaw CJ, Woo D, Adeoye O, Flaherty ML, Khatri P, et al (2012) Age at stroke: temporal trends in stroke incidence in a large, biracial population. Neurology 79, 1781-7.

24. Shumway-Cook A, Brauer S, Woollacott M (2000) Predicting the probability for falls in communitydwelling older adults using the timed up \& go test. Phys Ther 80, 896-903.

25. Plummer P, Eskes G, Wallace S, Giuffrida C, Fraas M, Campbell G, Clifton K, Skidmore ER (2013) Cognitive-motor interference during functional mobility after stroke: state of the science and implications for future research. Arch Phys Med Rehabil 94, 2565-74.

26. Woollacott M, Shumway-Cook A (2002) Attention and the control of posture and gait: a review of an emerging area of research. Gait Posture 16, 1-14.

27. Qu X (2014) Age-related cognitive task effects on gait characteristics: do different working memory components make a difference? J Neuroeng Rehabil $11,149$.

28. Salthouse TA, Babcock RL, Shaw RJ (1991) Effects of adult age on structural and operational capacities in working memory. Psychol Aging 6, 118-27.

29. Salthouse TA (1996) The processing-speed theory of adult age differences in cognition. Psychol Rev 103, 403-28.

30. Bensoussan L, Viton JM, Schieppati M, Collado H, Milhe de Bovis V, Mesure S, Delarque A (2007) Changes in postural control in hemiplegic patients after stroke performing a dual task. Arch Phys Med Rehabil 88, 1009-15.

31. Haggard P, Cockburn J, Cock J, Fordham C, Wade D (2000) Interference between gait and cognitive tasks in a rehabilitating neurological population. $J$ Neurol Neurosurg Psychiatry 69, 479-86.

32. Yogev-Seligmann G, Hausdorff JM, Giladi N (2012) Do we always prioritize balance when walking? towards an integrated model of task prioritization. Mov Disord 27, 765-70.

33. Plummer-D'Amato P, Altmann LJ (2012) Relationships between motor function and gait-related dualtask interference after stroke: a pilot study. Gait Posture 35, 170-2.

34. Lamontagne A, Fung J (2009) Gaze and postural reorientation in the control of locomotor steering 
after stroke. Neurorehabil Neural Repair 23, 256-66.

35. Reisman DS, Scholz JP, Schoner G (2002) Coordination underlying the control of whole body momentum during sit-to-stand. Gait Posture 15, 45-55.

36. Bonnyaud C, Pradon D, Bensmail D, Roche N (2015) Dynamic stability and risk of tripping during the timed up and go test in hemiparetic and healthy subjects. PLoS One 10, e0140317.

37. Dubost V, Kressig RW, Gonthier R, Herrmann FR, Aminian K, Najafi B, Beauchet O (2006) Relationships between dual-task related changes in stride velocity and stride time variability in healthy older adults. Hum Mov Sci 25, 372-82.

38. Olney SJ, Richards C (1996) Hemiparetic gait following stroke part I: characteristics. Gait Posture 4, 136-48. 